\title{
Maximum Entropy Production Is Not a Steady State Attractor for 2D Fluid Convection
}

\author{
Stuart Bartlett * and Nathaniel Virgo \\ Earth Life Science Institute, Tokyo Institute of Technology, 2-12-1-I7E-323 Ookayama, Meguro, Tokyo 152-8550, \\ Japan; nathanielvirgo@elsi.jp \\ * Correspondence: stuart.bartlett@elsi.jp; Tel.: +81-3-5734-2740
}

Academic Editor: Kevin H. Knuth

Received: 17 October 2016; Accepted: 28 November 2016; Published: 1 December 2016

\begin{abstract}
Multiple authors have claimed that the natural convection of a fluid is a process that exhibits maximum entropy production (MEP). However, almost all such investigations were limited to fixed temperature boundary conditions (BCs). It was found that under those conditions, the system tends to maximize its heat flux, and hence it was concluded that the MEP state is a dynamical attractor. However, since entropy production varies with heat flux and difference of inverse temperature, it is essential that any complete investigation of entropy production allows for variations in heat flux and temperature difference. Only then can we legitimately assess whether the MEP state is the most attractive. Our previous work made use of negative feedback BCs to explore this possibility. We found that the steady state of the system was far from the MEP state. For any system, entropy production can only be maximized subject to a finite set of physical and material constraints. In the case of our previous work, it was possible that the adopted set of fluid parameters were constraining the system in such a way that it was entirely prevented from reaching the MEP state. Hence, in the present work, we used a different set of boundary parameters, such that the steady states of the system were in the local vicinity of the MEP state. If MEP was indeed an attractor, relaxing those constraints of our previous work should have caused a discrete perturbation to the surface of steady state heat flux values near the value corresponding to MEP. We found no such perturbation, and hence no discernible attraction to the MEP state. Furthermore, systems with fixed flux BCs actually minimize their entropy production (relative to the alternative stable state, that of pure diffusive heat transport). This leads us to conclude that the principle of MEP is not an accurate indicator of which stable steady state a convective system will adopt. However, for all BCs considered, the quotient of heat flux and temperature difference $F / \Delta T$ - which is proportional to the dimensionless Nusselt number-does appear to be maximized.
\end{abstract}

Keywords: entropy production; convection; heat transfer; variational principle

\section{Introduction}

The natural convection of single phase fluids is an archetypal example of both non-linear dynamics and non-equilibrium thermodynamics. When heated from below and cooled from above, such fluids spontaneously structure themselves into a system of convection rolls, which tend to increase the rate of heat transport through the system (compared to that of diffusive transport alone). It has long been suggested - beginning with the pioneering work of Malkus [1-3] — that such phenomena express the system's attempts to maximize its rate of heat transport, subject to basic physical constraints. If the temperatures at the system's boundaries are held fixed, maximizing the rate of heat transport and maximizing entropy production become indistinguishable, since only the heat transport rate (to which entropy production rate is proportional) can vary. 
Further work since then (including theoretical and numerical studies [4,5]) has aimed to establish the scaling relation between the two primary dimensionless groups related to fluid convection: The Rayleigh number ( $\mathrm{Ra}$, a measure of thermal driving force), and the Nusselt number $(\mathrm{Nu}$, the ratio of total heat flux to diffusive heat flux). The majority of studies concerning convection made use of fixed temperature (perfectly conducting) boundaries. Such boundary conditions (BCs) are convenient for theoretical treatments, and can be implemented experimentally to a close approximation. A smaller number of works have also considered fixed heat flux boundaries [5-7], and the general result appears to be that the scaling relation between $\mathrm{Ra}$ and $\mathrm{Nu}$ is invariant to the type of $\mathrm{BC}$ for turbulent conditions. At low $\mathrm{Ra}$, fixed flux systems achieve a slightly augmented $\mathrm{Nu}$ compared to an equivalent fixed temperature system, due to the fact that the boundaries of the fixed flux system are not constrained to have horizontally uniform temperature profiles.

In previous work, we demonstrated (through the use of Lattice Boltzmann Model (LBM) simulations) that one can employ an alternative set of $\mathrm{BCs}$, where neither the boundary temperatures nor heat fluxes are fixed, and still observe the characteristic scaling relation between $\mathrm{Ra}$ and $\mathrm{Nu}$. These BCs are described as negative feedback BCs [8], since the heat flux into each boundary is constrained to be a decreasing function of the instantaneous boundary temperature. A defining characteristic of such BCs is that for any given set of boundary parameters, the system has the freedom to adjust its net heat flux between two well-defined bounds. The entropy production of the system, rather than changing monotonically between these two bounds, actually exhibits a single intermediate peak. It is thus an ideal test case for theories of maximum entropy production (MEP).

MEP is a variational principle which proposes that a non-equilibrium system with many degrees of freedom will adjust its internal dynamics such that its entropy production rate tends to a maximum, subject to any relevant physical constraints or conservation laws $[9,10]$. Negative feedback BCs were applied in the simple box models of Paltridge [11], which accurately predicted that atmospheric heat transport adheres to states of MEP. This early work catalysed a profusion of further investigations into the applicability, theoretical foundations, and consequences of MEP [9,12-20]. Indeed, it has been proposed that convecting fluids are a prime example of MEP [21-23]. In all of the aforementioned works, for the case of single phase fluid convection, only constant temperature BCs were considered. We would like to stress that entropy production should always be carefully distinguished from heat flux. Entropy production is the product of heat flux and the gradient of inverse temperature. Thus, statements about entropy production should include considerations of variable temperature difference, as well as variable heat flux. Alternatively, if an investigation only considers fixed temperature BCs, then it should be cautioned that only statements about the heat flux of those systems can be made.

Convecting fluids are characterized by a series of dynamical bifurcations as the thermal driving force is increased from 0 . The first-occurring at the so-called critical Rayleigh number-marks the point at which the linear diffusive state is unstable to perturbations, and the system cascades into a new configuration characterized by spatially structured velocity fields and convective heat transport between diffusive boundary layers. The primary approach of non-linear dynamics is to understand the nature of such bifurcations and why the system adopts a particular branch of steady states instead of alternative branches. In the case of the present work focussing on laminar convection, there are two steady states that the system could adopt—one in which the fluid remains motionless and heat moves by diffusion, and another in which there is sustained convective motion. Hence, variational principles such as MEP should predict which of these two discrete states the system will adopt (by way of comparing the two entropy production values). There is no continuous spectrum of stable states, simply the two branches extending beyond the first bifurcation. So, maximizing or minimizing entropy production in the present work refers to the discrete selection of the branch of states with the higher or lower entropy production.

In fact, the evidence from experimental and theoretical work compels us to conclude that convective systems maximize their entropy production under constant temperature BCs (maximum 
heat flux), but minimize their entropy production under constant flux BCs (minimum temperature difference) [24-27]. The case of minimum entropy production for flux-driven systems is simple to understand: below the critical Rayleigh number, in the linear diffusive regime, the temperature profile through the system is a simple linear extrapolation of the local temperature gradient (defined by the imposed heat flux) at the boundaries. Above the critical Rayleigh number, however, the steady state temperature difference drops below that of the diffusive regime, because the thermal impedance of the system is reduced when convection becomes an additional channel for heat transport. Thus, the system could produce more entropy by having the higher temperature difference of the diffusive regime. Instead, it bifurcates to a mode of convection with a characteristically lower temperature difference, which translates to a lower entropy production than the diffusive mode.

Recalling that the definition of the Nusselt number is $N u=F /(\chi \Delta T / H)$ (where $F$ is total heat flux, $\chi$ is thermal diffusivity, $\Delta T$ is temperature difference, and $H$ is vertical system dimension), it is clear that above the critical Rayleigh number, the entropy production will go down because we know that $N u>1$ and hence $F>(\chi \Delta T / H)$. Given that $F$ is fixed by the BCs, the temperature difference must have dropped below what it could be in the diffusive regime. Thus, the fact that the convective branch is stable beyond the bifurcation at the critical Ra implies that the system has chosen the steady state of lower entropy production (for fixed flux BCs).

In our previous numerical investigation, neither the heat flux nor the temperature difference of the system was fixed, since we employed negative feedback BCs [24]. We found that the system never evolved towards the MEP state. In fact, in all cases, the system's heat flux was far greater than that pertaining to MEP. It seemed that the steady state transport properties depended solely on the unique set of fluid and boundary parameters of that particular system. The scaling of the Rayleigh and Nusselt numbers was also shown to be identical to that of fixed BCs.

Given that the heat flux values that were exhibited in the previous work were significantly higher than that associated with MEP, it was possible that the boundary parameters we chose were acting as a binding constraint, holding the system away from the state of MEP. We were thus motivated to explore the possibility of systems with different boundary parameters, such that their steady states lie in the vicinity of the MEP peak. If MEP was indeed an attractor, and if our choice of parameters was a constraint preventing the system from reaching that attractor, then we would expect a discrete change in the distribution of steady state operating points of the system as the parameter constraints were relaxed. In other words, we would expect the density of steady state points to be higher near the MEP point.

In fact, it is straightforward to alter the boundary parameters such that the general position of the steady state along the heat flux axis is shifted (as will be shown in the following section). One can then more directly assess whether or not the MEP state represents a local attractor.

Thus, the objective of this work was to explore the steady states of fluid convection systems with negative feedback BCs in the vicinity of MEP (the heat flux value corresponding to MEP). We are thus building upon our previous analysis of entropy production in negative feedback fluid systems [24] and previous work on the transport properties of convective systems with fixed BCs $[5-7,27]$.

In the following section, we present the details of our model system and the various parameters and variables that characterise it. Since the LBM model employed here has been described in detail elsewhere $[24,25,28,29]$, we avoid an in-depth description in this paper. In Section 3 we present the results of our simulations. The paper concludes with a discussion of the implications of our findings for the wider relevance of MEP to fluid dynamical and heat transport systems.

\section{Model System}

The system in question is a vertically oriented two-dimensional fluid enclosed between solid boundaries at the top and bottom. Periodic BCs were used in the horizontal direction, and a fixed aspect ratio of 2:1 was adopted. The upper and lower boundaries receive inward heat fluxes $J_{i n, b}$ and $J_{i n, a}$ respectively, and are also able to radiate heat away. Following the example of the box models used 
by many previous authors $[9,11,16]$, the outward fluxes have the functional form $J_{\text {out }, i}=\beta T_{i}$, where $i \in\{a, b\}$ and $\beta$ is a parameter. Figure 1 shows a schematic of the system.

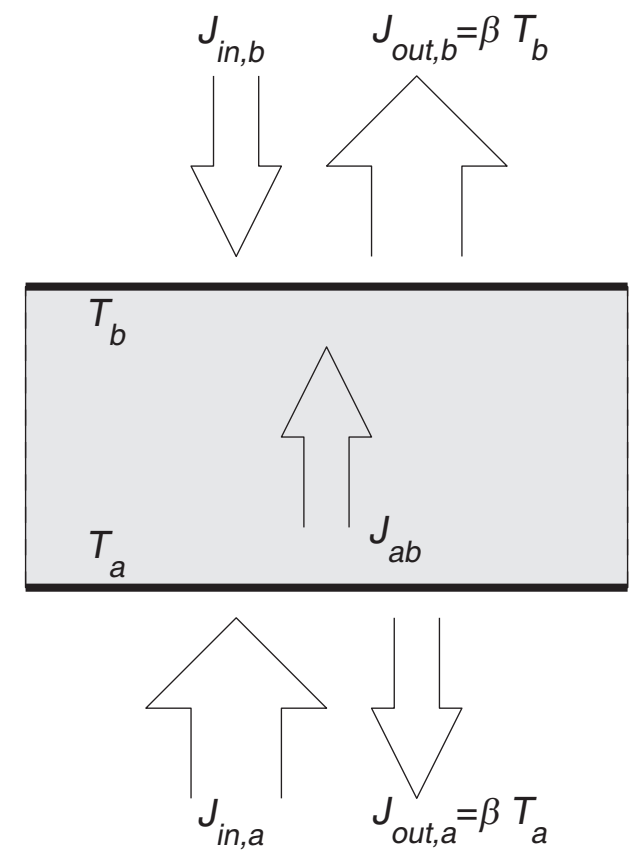

Figure 1. Model system diagram showing the various heat fluxes that comprise the boundary energy balances. Boundaries are periodic in the horizontal direction and enforce the no-slip velocity condition.

Heat flows through it by a combination of diffusion and convection. As mentioned in the previous section, simple box models with similar BCs have been used to approximate atmospheric heat transport [11,19,23], and have been explored analytically (e.g., [9]). Ignoring the internal details of the system and considering it as a black box that has reached a state of dynamic equilibrium, the energy balance at the two boundaries can be written as

$$
\begin{gathered}
J_{i n, a}-J_{a b}-\beta T_{a}=0 \\
J_{i n, b}+J_{a b}-\beta T_{b}=0 .
\end{gathered}
$$

Summing these two equations yields

$$
J_{i n, a}+J_{i n, b}-\beta\left(T_{a}+T_{b}\right)=0
$$

If we write the two temperatures in the form $T_{a}=T_{0}+\Delta T / 2$ and $T_{b}=T_{0}-\Delta T / 2$, we can use Equation (3) to find an expression for the system's average temperature $T_{0}=\left(T_{a}+T_{b}\right) / 2$,

$$
T_{0}=\frac{J_{i n, a}+J_{i n, b}}{2 \beta} .
$$

To find an expression in terms of $\Delta T$, we can subtract Equation (2) from Equation (1):

$$
J_{i n, a}-J_{i n, b}-2 J_{a b}=\beta \Delta T \text {. }
$$

As mentioned previously, we see that the BCs force the heat flux to be a decreasing function of the temperature difference. Furthermore, the constraint of Equation (5) means that the system has only one macroscopic degree of freedom (despite the fact that neither the temperature difference nor the heat flux are explicitly fixed). To determine the steady state properties exactly, we would require an additional 
constraint-for example, the relationship between $J_{a b}$ and $\Delta T$. Such a relationship would be linear for diffusive heat transport. For convective transport, it is generally not known. Various empirical scaling laws have been observed, and theoretical studies have also produced candidate expressions. However, at present, it is still not clear whether a universally valid force-flux relationship exists $[4,5,30]$.

How the system will choose its steady state is not entirely clear, hence the potential utility of a selection mechanism such as MEP. We shall now see how a characteristic peak in entropy production emerges from the boundary energy balance expressions. First let us consider the extrema of $J_{a b}$ and $\Delta T$, making use of Equation (5). In the case of zero flux through the fluid, $J_{a b}=0$ and the temperature difference is maximized: $\Delta T_{\max }=\left(J_{i n, a}-J_{i n, b}\right) / \beta=\Delta J_{i n} / \beta$. Conversely, if the temperature difference becomes negligible $(\Delta T=0)$, the heat flux is maximized: $J_{a b_{\max }}=\Delta J_{i n} / 2$. The two maxima can be used to calculate dimensionless versions of the heat flux and temperature difference: $\overline{J_{a b}}=J_{a b} / J_{a b_{\max }}$ and $\overline{\Delta T}=\Delta T / \Delta T_{\max }$. We can now find the entropy production as a function of a single dimensionless variable of the system. Noting the dimensions of $\beta$, the dimensionless entropy production can be defined as $\bar{\sigma}=\sigma / \beta$ :

$$
\bar{\sigma}=\frac{J_{a b}}{\beta}\left(\frac{1}{T_{b}}-\frac{1}{T_{a}}\right),
$$

which, after some manipulation, becomes

$$
\bar{\sigma}=\frac{2 \overline{J_{a b}} \Delta J_{i n}^{2}\left(1-\overline{J_{a b}}\right)}{\left(J_{i n, a}+J_{i n, b}\right)^{2}-\left(\Delta J_{i n}\left[1-\overline{J_{a b}}\right]\right)^{2}} .
$$

We see that the dimensionless entropy production only depends upon the normalized variable $\overline{J_{a b}}$ and the inward flux parameters $J_{i n_{a}}$ and $J_{i n_{b}}$. Compared to our previous study, in this work we used a new value for the parameter $\beta$, and the other parameters remain unchanged $\left(J_{i n_{a}}=0.1, J_{i n_{b}}=0.01, \beta=1.6\right.$ for the present work). MEP corresponds to maximizing $\bar{\sigma}$ with respect to $\overline{J_{a b}}$. This is displayed graphically in Figure 2 .

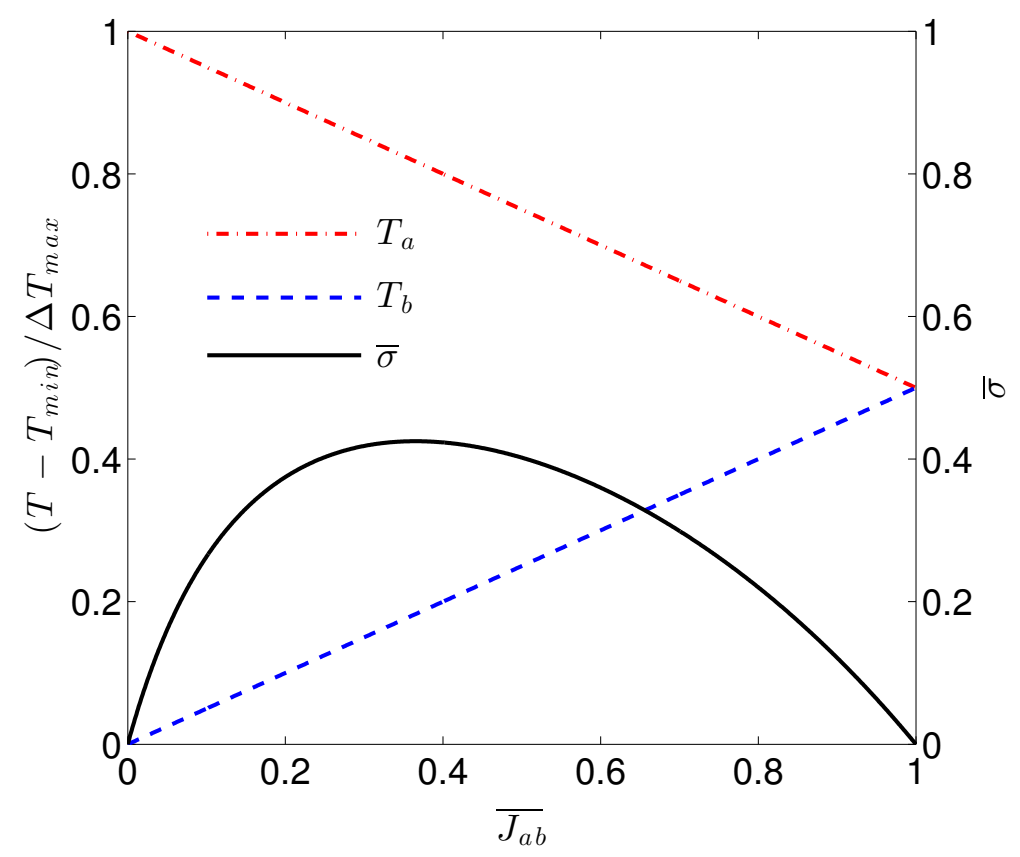

Figure 2. Macroscopic transport properties of the model system. The boundary temperatures $T_{a}$ (red dot-dash line) and $T_{b}$ (blue dashed line) and dimensionless entropy production $\bar{\sigma}$ (solid black line) are plotted as a function of the normalized heat flux $\overline{J_{a b}}$. 
We see that the entropy production peak emerges from the compromise of increasing heat flux vs. decreasing temperature gradient.

To simulate the fluid dynamic and thermodynamic behaviour of such a system, we employed a thermal LBM, first introduced by He et al. [28] and simplified by Peng et al. [29]. Details of the exact implementation of negative feedback BCs can be found in Bartlett and Bullock [24], Bartlett [25]. The numerical parameters of each simulation carried out are given in Table 1.

Table 1. Mean Rayleigh numbers for each Lattice Boltzmann Model parameter combination (velocity distribution relaxation parameter $\tau_{v}$ and internal energy distribution relaxation parameter $\tau_{c}$ ).

\begin{tabular}{|c|c|c|c|c|c|c|c|c|c|}
\hline & & \multicolumn{8}{|c|}{$\tau_{v}$} \\
\hline & & 0.70 & 0.75 & 0.80 & 0.85 & 0.90 & 0.95 & 1.00 & 1.05 \\
\hline \multirow{6}{*}{$\tau_{c}$} & 0.55 & $4.7 \times 10^{4}$ & $3.9 \times 10^{4}$ & $3.2 \times 10^{4}$ & $2.8 \times 10^{4}$ & $2.5 \times 10^{4}$ & $2.2 \times 10^{4}$ & $2.0 \times 10^{4}$ & $1.8 \times 10^{4}$ \\
\hline & 0.60 & $2.1 \times 10^{4}$ & $1.7 \times 10^{4}$ & $1.4 \times 10^{4}$ & $1.2 \times 10^{4}$ & $1.1 \times 10^{4}$ & $9.7 \times 10^{3}$ & $8.8 \times 10^{3}$ & $8.1 \times 10^{3}$ \\
\hline & 0.65 & $1.2 \times 10^{4}$ & $1.0 \times 10^{4}$ & $8.5 \times 10^{3}$ & $7.4 \times 10^{3}$ & $6.6 \times 10^{3}$ & $6.0 \times 10^{3}$ & $5.4 \times 10^{3}$ & $5.0 \times 10^{3}$ \\
\hline & 0.70 & $8.5 \times 10^{3}$ & $7.0 \times 10^{3}$ & $6.0 \times 10^{3}$ & $5.2 \times 10^{3}$ & $4.7 \times 10^{3}$ & $4.2 \times 10^{3}$ & $3.8 \times 10^{3}$ & $3.5 \times 10^{3}$ \\
\hline & 0.75 & $7.0 \times 10^{3}$ & $5.3 \times 10^{3}$ & $4.5 \times 10^{3}$ & $4.0 \times 10^{3}$ & $3.6 \times 10^{3}$ & $3.2 \times 10^{3}$ & $3.0 \times 10^{3}$ & $2.8 \times 10^{3}$ \\
\hline & 0.80 & $5.1 \times 10^{3}$ & $4.2 \times 10^{3}$ & $3.6 \times 10^{3}$ & $3.2 \times 10^{3}$ & $2.9 \times 10^{3}$ & $2.6 \times 10^{3}$ & $2.4 \times 10^{3}$ & $2.3 \times 10^{3}$ \\
\hline
\end{tabular}

The relaxation parameter $\tau_{v}$ defines the fluid viscosity $v$ :

$$
v=\frac{1}{3}\left(\tau_{v}-\frac{1}{2}\right) c^{2}
$$

where $c$ is the lattice spacing. The thermal diffusivity $\chi$ is controlled analogously:

$$
\chi=\frac{2}{3}\left(\tau_{c}-\frac{1}{2}\right) c^{2}
$$

The values within the body of Table 1 are the steady state Rayleigh numbers exhibited by each combination of $\tau_{v}$ and $\tau_{c}$. As can be seen from these values, we restricted the simulations to laminar flows (the simulation domain size was fixed at $W=400$, and the height was $H=200$ lattice cells). In previous work, we experimented with high Rayleigh number flows (up to $R a=10^{8}$ ) and found that more turbulent conditions had no effect on whether the system was attracted to states of MEP. This, combined with the large computational burden of turbulent systems, led us to restrict ourselves to laminar flows in the present work.

We also used the uni-temp BC, wherein the incoming heat flux at each time step is distributed across boundary grid cells, such that the boundary temperature remains horizontally uniform. Note that the use of BCs in which the boundary temperature is free to be non-uniform do not exhibit significantly different results (see Bartlett and Bullock [24], Bartlett [25] for details). All simulations had the same boundary parameters.

If MEP was indeed an attractor for these systems, and some combination of the fluid viscosity and thermal diffusivity was a constraining factor preventing MEP from being expressed, we should expect a discrete and abrupt adherence to the heat flux value corresponding to MEP as the two fluid parameters are varied and the steady state of the system approaches that of MEP.

\section{Results}

The results of our simulations are summarized in Figure 3, which shows the normalized steady state heat flux $\overline{J_{a b}}$ as a function of the two fluid parameters (the fluid viscosity $v$ and thermal diffusivity $\chi$ ). A horizontal plane representing the heat flux value corresponding to the MEP state is also shown. 
We see that the heat flux clearly increases with smaller viscosities and higher thermal diffusivities. This result aligns with physical intuition: lower viscosities allow a greater degree of convective heat transport, and higher thermal diffusivities allow a larger diffusive heat flux within the two boundary layers of the flow. The heat transport characteristics are exactly what we would expect. However, the steady state seems to be in no way influenced by MEP, since the surface of steady state heat flux values passes straight through the MEP plane without any kind of perturbation (e.g., a point of inflection or change in curvature of the surface).

It was also found that the scaling of $\mathrm{Nu}$ as a function of Ra was again identical to that of fixed BCs. This characteristic scaling behaviour is illustrated in Figure 3 of Bartlett and Bullock [24], and other works in which constant BCs were used (e.g., Figure 4 of [5]).

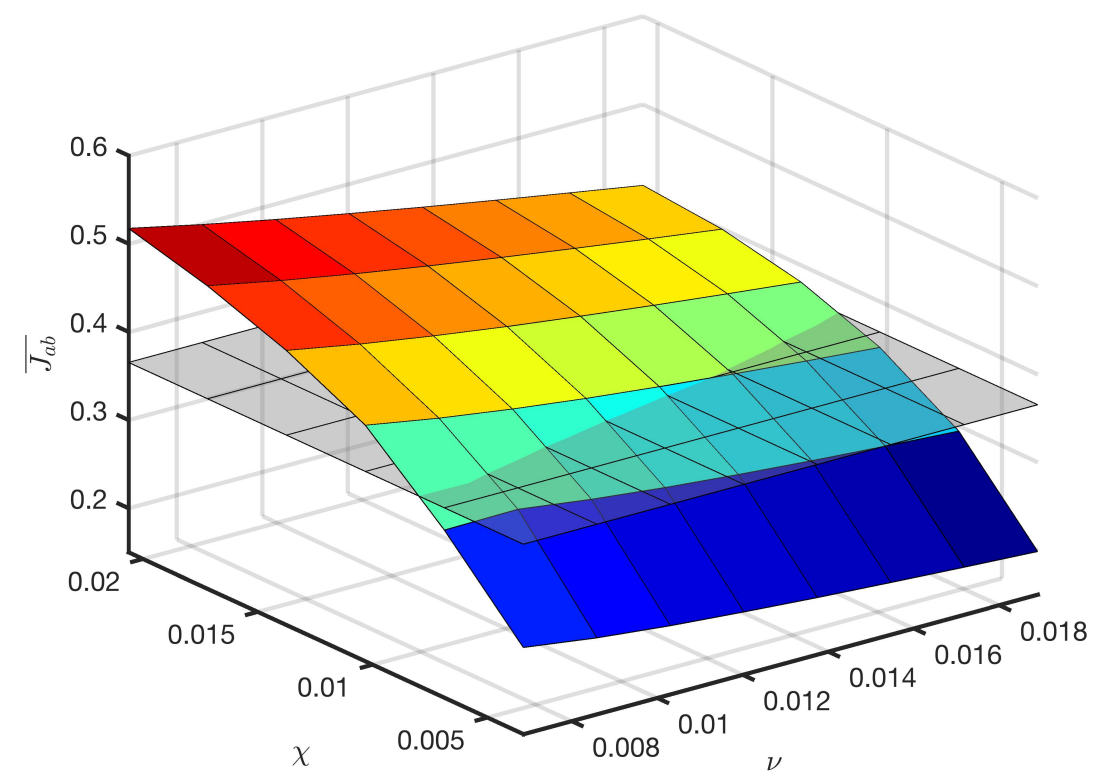

Figure 3. Normalized steady state heat flux $\overline{J_{a b}}$ as a function of fluid viscosity $v$ and thermal diffusivity $\chi$. The grey plane represents the heat flux value corresponding to maximum entropy production (MEP).

\section{Discussion}

In our previous work, we showed that the use of negative feedback BCs had no influence on the scaling behaviour of the dimensionless parameters $\mathrm{Ra}$ and $\mathrm{Nu}$ for single phase fluid convection. With the parameter set used in that investigation, simulated heat flux values were far higher than the value corresponding to MEP. In this paper, we modified the boundary parameters such that the steady states of the system lay in the vicinity of the MEP state. This was motivated by the possibility that our previous parameter choices constituted a preventive constraint on the system. Since entropy production can only be maximized subject to constraints, we were compelled to assess whether relaxing the parameter choice constraint might induce a qualitative change in the system's choice of steady state and allow it to adhere to MEP.

The results of our simulations support the conclusions of our previous work that for single phase fluid convection, MEP does not represent a steady state attractor. We see no reason why this system should be sensitive to its net thermal entropy production, given that it depends only upon boundary parameters, rather than both boundary and fluid parameters. As we have shown, different sets of fluid parameters produce different steady states, and there is no increase in the density of states as the parameters allow the system to approach MEP.

What remains to be elucidated is the connection between our negative result and previous positive results which showed that atmospheric heat transport adheres to MEP $[9,11,16,18,19]$. It could be the 
case that non-equilibrium systems need a threshold number of degrees of freedom in order to have the ability to express MEP, and the current 2D single-phase system is simply not complex enough (its behaviour is not significantly more complex than a dynamical system with two degrees of freedom, despite being a fluid comprised of a very large number of microscopic degrees of freedom).

It would be desirable to perform similar numerical experiments in the turbulent regime. This would require either a modified LBM algorithm (e.g., [31]), very high resolution grids, or simply a different simulation technique. However, there is no reason to believe that the conclusion of this and our previous work would be altered. The steady states of all systems analyzed correspond to the point of intersection between the force-flux curve of the fluid and the force-flux curve of the BCs. We believe that this trend of the system finding the single steady state compatible with both the fluid force-flux relationship and the BC force-flux relationship would continue to higher, turbulent Rayleigh numbers.

The question of a universal extremum principle for nonequilibrium systems remains very much open. We believe it is clear that MEP cannot be interpreted as a general principle of nature. However, there is a combination of variables which is always maximized for all three BCs considered by this and previous work, and this will be discussed in the final section.

\section{Further Work}

Single phase fluid convection seems to have a single force-flux curve, from which the system never deviates (the oft alluded to scaling relation between $\mathrm{Ra}$ and $\mathrm{Nu}$ ). Physically, how can we interpret this force-flux curve? What does it represent? As discussed previously, If we constrain a system to fixed boundary temperatures, it will transport as much heat between the boundaries as it can, subject to the limits of finite viscosity and thermal diffusivity. In the case of fixed flux systems, the lowest possible driving gradient will emerge, such that the imposed flux can be maintained. Thus, the variable maximized in both cases (and also in the case of negative feedback BCs) is proportional to $F / \Delta T$, suggesting the Nusselt number, $N u=F /(\chi \Delta T / H)$, as a possible maximization target of any convective system. We are currently experimenting with further novel BCs to explore whether this possibility is universally applicable.

Acknowledgments: This work was supported by an EPSRC Doctoral Training Centre grant (EP/G03690X/1), and the Earth Life Science Institute Origins Network (EON) Postdoctoral Fellowship Program.

Author Contributions: This paper is an extension of work carried out during S.B.'s PhD. S.B. constructed and ran the numerical simulations, generated the figures and wrote the text. N.V. suggested the idea of displaying the system's steady states passing through the MEP plane, and provided important feedback and advice during the early stages of the work and the writing of the manuscript.

Conflicts of Interest: The authors declare no conflict of interest.

\section{Abbreviations}

The following abbreviations are used in this manuscript:

BC Boundary condition

LBM Lattice Boltzmann Model

MEP Maximum Entropy Production

\section{References}

1. Malkus, W.V.R. Discrete transitions in turbulent convection. Proc. R. Soc. Lond. Ser. A 1954, 225, 185-195.

2. Malkus, W.V.R. The heat transport and spectrum of thermal turbulence. Proc. R. Soc. Lond. Ser. A 1954, 225, 196-212.

3. Malkus, W.V.R.; Veronis, G. Finite amplitude cellular convection. J. Fluid Mech. 1958, 4, 225-260.

4. Ahlers, G.; Grossmann, S.; Lohse, D. Heat transfer and large scale dynamics in turbulent Rayleigh-Bénard convection. Rev. Mod. Phys. 2009, 81, 503-537.

5. Johnston, H.; Doering, C.R. Comparison of turbulent thermal convection between conditions of constant temperature and constant flux. Phys. Rev. Lett. 2009, 102, 064501.

6. Otero, J.; Wittenberg, R.W.; Worthin, R.A.; Doering, C.R. Bounds on Rayleigh-Bénard convection with an imposed heat flux. J. Fluid Mech. 2002, 473, 191-199. 
7. Verzicco, R.; Sreenivasan, K.R. A comparison of turbulent thermal convection between conditions of constant temperature and constant heat flux. J. Fluid Mech. 2008, 595, 203-219.

8. Virgo, N.D. Thermodynamics and the Structure of Living Systems. Ph.D. Thesis, University of Sussex, Brighton, UK, May 2011.

9. Kleidon, A. Nonequilibrium thermodynamics and maximum entropy production in the Earth system. Naturwissenschaften 2009, 96, 653-677.

10. Martyushev, L.; Seleznev, V. Maximum entropy production principle in physics, chemistry and biology. Phys. Rep. 2006, 426, 1-45.

11. Paltridge, G.W. The steady-state format of global climate. Q. J. R. Meteorol. Soc. 1978, 104, 927-945.

12. Dewar, R. Information theory explanation of the fluctuation theorem, maximum entropy production and self-organized criticality in non-equilibrium stationary states. J. Phys. A Math. Gen. 2003, 36, 631.

13. Dewar, R.C. Maximum entropy production as an inference algorithm that translates physical assumptions into macroscopic predictions: Don't shoot the messenger. Entropy 2009, 11, 931-944.

14. Dewar, R.C. Maximum entropy production and plant optimization theories. Philos. Trans. R. Soc. Lond. Ser. B 2010, 365, 1429-1435.

15. Dyke, J.; Kleidon, A. The maximum entropy production principle: Its theoretical foundations and applications to the Earth system. Entropy 2010, 12, 613-630.

16. Kleidon, A. A basic introduction to the thermodynamics of the Earth system far from equilibrium and maximum entropy production. Philos. Trans. R. Soc. Lond. Ser. B 2010, 365, 1303-1315.

17. Kleidon, A.; Malhi, Y.; Cox, P.M. Maximum entropy production in environmental and ecological systems. Philos. Trans. R. Soc. Lond. Ser. B 2010, 365, 1297-1302.

18. O’Brien, D.M.; Stephens, G.L. Entropy and climate. II: Simple models. Q. J. R. Meteorolog. Soc. 1995, 121, 1773-1796.

19. Ozawa, H.; Ohmura, A.; Lorenz, R.D.; Pujol, T. The second law of thermodynamics and the global climate system: A review of the maximum entropy production principle. Rev. Geophys. 2003, 41, doi:10.1029/2002RG000113.

20. Vallino, J.J. Ecosystem biogeochemistry considered as a distributed metabolic network ordered by maximum entropy production. Philos. Trans. R. Soc. Lond. Ser. B 2010, 365, 1417-1427.

21. Bradford, R. An investigation into the maximum entropy production principle in chaotic Rayleigh Benard convection. Physica A 2013, 392, 6273-6283.

22. Kita, T. Principle of maximum entropy applied to Rayleigh-Bénard convection. J. Phys. Soc. Jpn. 2006, 75, 124005, doi:10.1143/JPSJ.75.124005.

23. Ozawa, H.; Shimokawa, S.; Sakuma, H. Thermodynamics of fluid turbulence: A unified approach to the maximum transport properties. Phys. Rev. E 2001, 64, 026303, doi:10.1103/PhysRevE.64.026303.

24. Bartlett, S.; Bullock, S. Natural convection of a two-dimensional Boussinesq fluid does not maximize entropy production. Phys. Rev. E 2014, 90, 023014, doi:10.1103/PhysRevE.90.023014.

25. Bartlett, S.J. Why is Life? An Assessment of the Thermodynamic Properties of Dissipative, Pattern-forming Systems. Ph.D. Thesis, University of Southampton, Southampton, UK, July 2014.

26. Kawazura, Y.; Yoshida, Z. Entropy production rate in a flux-driven self-organizing system. Phys. Rev. E 2010, 82, 066403, doi:10.1103/PhysRevE.82.066403.

27. Kawazura, Y.; Yoshida, Z. Comparison of entropy production rates in two different types of self-organized flows: Bénard convection and zonal flow. Phys. Plasmas 2012, 19, 012305, doi:10.1063/1.3675854.

28. He, X.; Chen, S.; Doolen, G.D. A novel thermal model for the lattice Boltzmann method in incompressible limit. J. Comput. Phys. 1998, 146, 282-300.

29. Peng, Y.; Shu, C.; Chew, Y.T. Simplified thermal lattice Boltzmann model for incompressible thermal flows. Phys. Rev. E 2003, 68, 026701, doi:10.1103/PhysRevE.68.026701

30. Grossmann, S.; Lohse, D. Scaling in thermal convection: a unifying theory. J. Fluid Mech. 2000, 407, 27-56.

31. Dixit, H.; Babu, V. Simulation of high Rayleigh number natural convection in a square cavity using the lattice Boltzmann method. Int. J. Heat Mass Transf. 2006, 49, 727-739.

(C) 2016 by the authors; licensee MDPI, Basel, Switzerland. This article is an open access article distributed under the terms and conditions of the Creative Commons Attribution (CC-BY) license (http:/ / creativecommons.org/licenses/by/4.0/). 